\title{
A structure theorem of Dirac-harmonic maps between spheres
}

\section{Ling Yang}

Received: 18 June 2008 / Accepted: 20 October 2008 / Published online: 11 November 2008

(C) The Author(s) 2008. This article is published with open access at Springerlink.com

\begin{abstract}
For an arbitrary Dirac-harmonic map $(\phi, \psi)$ between compact oriented Riemannian surfaces, we shall study the zeros of $|\psi|$. With the aid of Bochner-type formulas, we explore the relationship between the order of the zeros of $|\psi|$ and the genus of $M$ and $N$. On the basis, we could clarify all of non-trivial Dirac-harmonic maps from $S^{2}$ to $S^{2}$.
\end{abstract}

Mathematics Subject Classification (2000) $\quad 58 \mathrm{E} 20 \cdot 53 \mathrm{C} 27$

\section{Introduction}

Let $(M, h)$ be an $m$-dimensional Riemannian spin manifold; Spin $M$ denotes the Spin-bundle on $M$, and $\eta: \operatorname{Spin} M \rightarrow S O M$ is the bundle map, where $S O M$ denotes the tangent orthonormal frame bundle on $M$. Denote by $\Sigma M$ the spinor bundle associated to Spin $M$, i.e. $\Sigma M=\operatorname{Spin} M \times_{\rho} \Sigma_{m}$, where $\rho: \operatorname{Spin}_{m} \rightarrow \Sigma_{m}$ is the standard representation. On $\Sigma M$ we can choose an Hermitian product $\langle$, $\rangle$, such that

$$
\langle X \cdot \psi, \xi\rangle=-\langle\psi, X \cdot \xi\rangle \quad X \in \Gamma(T M), \psi, \xi \in \Gamma(\Sigma M) .
$$

Here

$$
\mathfrak{m}: X \otimes \psi \mapsto X \cdot \psi
$$

is the Clifford multiplication. There is a connection on $\Sigma M$ induced by the Levi-Civita connection of $S O M$; denote it by $\nabla$; and it is well known that $\nabla$ is compatible with $\langle$,$\rangle . Let$ $\phi$ be a smooth map from $M$ to another Riemannian manifold $(N, g)$ of dimension $n \geq 2$. Denote by $\phi^{-1} T N$ the pull-back bundle of $T N$ and by $\Sigma M \otimes \phi^{-1} T N$ the twisted bundle. On it there is a metric induced from those on $\Sigma M$ and $\phi^{-1} T N$. Similarly we have a natural connection $\tilde{\nabla}$ on $\Sigma M \otimes \phi^{-1} T N$ induced from those on $\Sigma M$ and $\phi^{-1} T N$. Based on it, we

L. Yang $(\bowtie)$

Max-Planck Institute for Mathematics in the Sciences, Inselstr. 22-26, 04103 Leipzig, Germany

e-mail: lingyang@mis.mpg.de 
can define the Dirac operator along the map $\phi$ by

$$
\not D \psi=\mathfrak{m} \circ \tilde{\nabla} \psi .
$$

Here $\psi$ is a smooth section of $\Sigma M \otimes \phi^{-1} T N$.

In Chen et al. [1], introduced a functional that couples the non-linear sigma model with a spinor field:

$$
L(\phi, \psi)=\int_{M}\left[|d \phi|^{2}+\langle\psi, \not D \psi\rangle\right] * 1
$$

The critical points of the functional are called Dirac-harmonic maps. In the paper, some geometric and analytic aspects of such maps were studied, especially a removable singularity theorem was established. Later in $[2,3,9,10]$, another geometric and analytic properties of Dirac-harmonic maps were studied.

Obviously there are two type of trivial Dirac-harmonic maps. One is $(\phi, 0)$, where $\phi$ is a harmonic map, and another is $(y, \psi)$, where $y$ is a point in $N$ viewed as a constant map, $\psi$ is a harmonic spinor. In [1], the authors constructed non-trivial Dirac-harmonic maps $(\phi, \psi)$ from $S^{2}$ to $S^{2}$, where $\phi$ is a (possible branched) conformal map, $\psi$ could be written in the form

$$
\psi=e_{\alpha} \cdot \Psi \otimes \phi_{*}\left(e_{\alpha}\right),
$$

$\left\{e_{\alpha}: \alpha=1,2\right\}$ is a local orthonormal frame field on $S^{2}$, and $\Psi$ is a twistor spinor. It is natural to ask whether there exists another form of Dirac-harmonic maps from $S^{2}$ to $S^{2}$. And furthermore, is there a Dirac-harmonic map $(\phi, \psi)$ such that $\phi$ is not a harmonic map?

In the theory of harmonic maps between two compact Riemannian surfaces, Bochner formulas of $\log |\partial u|$ and $\log |\bar{\partial} u|$ play an important role ( $u$ denotes a harmonic map). From it several interesting formulas easily follow, which tell us the relationship between the order of the zeros of $\log |\partial u|$ and $\log |\bar{\partial} u|$ and the genus of $M$ and $N$; and moreover we can obtain some uniqueness theorems and non-existence theorems (see [7, Chapter I]). This phenomenon motives us to study the zeros of $|\psi|$.

Now we give a brief outline of the paper. In Sect. 2, the subjects we study are general Dirac-harmonic maps. In the viewpoint that $\Sigma M \otimes \phi^{-1} T N=\Sigma M \otimes\left(\phi^{-1} T N\right)^{\mathbb{C}}, \psi$ could be written as $\psi=\psi^{j} \otimes W_{j}$, where $\left\{W_{1}, \ldots, W_{n}\right\}$ is a local complex tangent frame field on $N$; and we derive the Euler-Lagrange equation of $L$ by using the above denotation. In Sect. 3, we assume $M$ and $N$ to be oriented Riemannian surfaces; the equations of harmonic spinor $\psi$ along $\phi$ in the local complex coordinates are derived, which imply that the zeros of $\left|\pi_{1}^{+}(\psi)\right|$ are isolated, unless $\left|\pi_{1}^{+}(\psi)\right|$ is identically zero, so are $\left|\pi_{0}^{+}(\psi)\right|,\left|\pi_{1}^{-}(\psi)\right|$ and $\left|\pi_{0}^{-}(\psi)\right|$. (Here the definition of $\pi_{1}^{+}, \pi_{0}^{+}, \pi_{1}^{-}, \pi_{0}^{-}$is introduced in Sect. 3.) In Sects. 4 and 5, under the further assumption that $M$ and $N$ are both compact, we derive several Bochner formulas of $\log \left|\pi_{1}^{+}(\psi)\right|, \log \left|\pi_{0}^{+}(\psi)\right|, \log \left|\pi_{1}^{-}(\psi)\right|, \log \left|\pi_{0}^{-}(\psi)\right|$ on the basis of Weitzenböck-type formulas of $\psi$ and furthermore give the proof of main theorems as follows, including a structure theorem of Dirac-harmonic maps from $S^{2}$ to $S^{2}$. (In the process, it is necessary to use the results in Sects. 2 and 3).

Theorem 1.1 $M$ and $N$ are both compact oriented Riemannian surfaces, and $(\phi, \psi)$ is a Dirac-harmonic map from $M$ to $N$. If $g_{M}=0$ or $\left|g_{M}-1\right|<|\operatorname{deg}(\phi)|\left|2 g_{N}-2\right|$, then $\phi$ has to be a harmonic map.

Theorem 1.2 If $M=N=S^{2}$ equipped with arbitrary metric, $(\phi, \psi)$ is a non-trivial Diracharmonic map from $M$ to $N$, then $\phi$ has to be holomorphic or anti-holomorphic, $\psi$ could be 
written in the form

$$
\psi=e_{\alpha} \cdot \Psi \otimes \phi_{*}\left(e_{\alpha}\right),
$$

where $\Psi$ is a twistor spinor (possibly with isolated singularities).

Please note that here and in the sequel we use the summation convention and agree the range of indices:

$$
1 \leq i, j, k \leq n ; \quad 1 \leq \alpha, \beta \leq m .
$$

We refer to [4-6] for more background material on spin structures and Dirac operators and to $[7,8]$ for more background material on harmonic maps.

\section{Euler-Lagrange equations of Dirac-harmonic maps}

Denote the complexification of $\phi^{-1} T N$ by $\left(\phi^{-1} T N\right)^{\mathbb{C}}$. Obviously $\Sigma M \otimes \phi^{-1} T N \subset \Sigma M \otimes$ $\left(\phi^{-1} T N\right)^{\mathbb{C}}$. On the other hand, for any $\psi \in \Sigma M, X+\sqrt{-1} Y \in\left(\phi^{-1} T N\right)^{\mathbb{C}}$ (here $X, Y \in$ $\left.\phi^{-1} T N\right)$,

$$
\psi \otimes(X+\sqrt{-1} Y)=\psi \otimes X+\sqrt{-1} \psi \otimes Y \in \Sigma M \otimes \phi^{-1} T N
$$

which implies $\Sigma M \otimes\left(\phi^{-1} T N\right)^{\mathbb{C}} \subset \Sigma M \otimes \phi^{-1} T N$. Hence $\Sigma M \otimes\left(\phi^{-1} T N\right)^{\mathbb{C}}=\Sigma M \otimes$ $\phi^{-1} T N$. The pull-back metric $\phi^{-1} g$ on $\phi^{-1} T N$ could be naturally extended to a Hermitian product on $\left(\phi^{-1} T N\right)^{\mathbb{C}}$; and there is a natural Hermitian product on $\Sigma M \otimes\left(\phi^{-1} T N\right)^{\mathbb{C}}$ induced from those on $\Sigma M$ and $\left(\phi^{-1} T N\right)^{\mathbb{C}}$, which is also denoted by $\langle$,$\rangle .$

For each point $x \in M$, we can choose $\left\{W_{i} \in \Gamma\left((T U)^{\mathbb{C}}\right): 1 \leq i \leq n\right\}$, where $U$ is a neighborhood of $\phi(x)$, such that

$$
\left(T_{\phi(y)} N\right)^{\mathbb{C}}=\bigoplus_{i=1}^{n} \mathbb{C} W_{i}(\phi(y)) \quad y \in \phi^{-1}(U),
$$

then on $\phi^{-1}(U), \psi$ could be expressed by

$$
\psi(y)=\psi_{j}(y) \otimes W_{i}(\phi(y)),
$$

where $\psi_{1}, \ldots, \psi_{n} \in \Gamma\left(\Sigma\left(\phi^{-1}(U)\right)\right.$. We shall derive the Euler-Lagrange equations for $L$ by using the above denotation.

Proposition 2.1 Let $\left\{e_{\alpha}: 1 \leq \alpha \leq m\right\}$ be a local tangent orthonormal frame field, Then the Euler-Lagrange equations for $L$ are

$$
\begin{aligned}
\not D \psi & =0 \\
\tau(\phi) & =-\left\langle\psi^{j}, e_{\alpha} \cdot \psi^{k}\right\rangle R_{W_{j}, W_{k}}^{N} \phi_{*}\left(e_{\alpha}\right) .
\end{aligned}
$$

Proof At first, we consider a family of $\psi_{t}$ with $\frac{d \psi_{t}}{d t}=\eta$ at $t=0$ and fix $\phi$. Since $\not D$ is formally self-adjoint (see [1]), we have

$$
\begin{aligned}
\left.\frac{d L}{d t}\right|_{t=0} & =\int_{M}\langle\eta, \not D \psi\rangle+\langle\psi, \not D \eta\rangle=\int_{M}\langle\eta, \not D \psi\rangle+\langle\not D \psi, \eta\rangle \\
& =2 \int_{M} \operatorname{Re}\langle\eta, \not D \psi\rangle .
\end{aligned}
$$

Since $\eta$ could be chosen arbitrarily, (2.3) is easily followed. 
Now we consider a variation $\phi_{t}(t \in(-\varepsilon, \varepsilon))$ of $\phi$ such that $\phi_{t}=\phi$ outside a compact set $K \subset \subset \phi^{-1}(U)$ and $\phi_{t}(K) \subset U$; denote $\psi(y)=\psi^{j}(y) \otimes W_{j}(\phi(y))$ for each $y \in U$, then we define $\psi_{t}(y)=\psi^{j}(y) \otimes W_{j}\left(\phi_{t}(y)\right)$. Denote $\xi=\left.\frac{d \phi_{t}}{d t}\right|_{t=0}$. Obviously

$$
\left.\frac{d L}{d t}\right|_{t=0}=\left.\int_{M} \frac{d}{d t}\right|_{t=0}\left|d \phi_{t}\right|^{2}+\left.\int_{M} \frac{d}{d t}\right|_{t=0}\langle\psi, \not D \psi\rangle=I+I I,
$$

and

$$
I=-2 \int_{M}\langle\xi, \tau(\phi)\rangle .
$$

Here $\tau(\phi)$ denotes the tension field of $\phi$. Since $\psi=\psi^{j} \otimes W_{j}$, we have

$$
\not D \psi=\not \partial \psi^{k} \otimes W_{k}+e_{\alpha} \cdot \psi^{k} \otimes \nabla_{e_{\alpha}} W_{k},
$$

where $\not \partial$ denotes the usual Dirac operator. Then

$$
\begin{aligned}
\left.\frac{d}{d t}\right|_{t=0} \not D \psi & =\not \partial \psi^{k} \otimes \nabla_{\frac{\partial}{\partial t}} W_{k}+e_{\alpha} \cdot \psi^{k} \otimes \nabla_{\frac{\partial}{\partial t}} \nabla_{e_{\alpha}} W_{k} \\
& =e_{\alpha} \cdot \nabla_{e_{\alpha}} \psi^{k} \otimes \nabla_{\frac{\partial}{\partial t}} W_{k}+e_{\alpha} \cdot \psi^{k} \otimes \nabla_{e_{\alpha}} \nabla_{\frac{\partial}{\partial t}} W_{k}+e_{\alpha} \cdot \psi^{k} \otimes R_{e_{\alpha}, \frac{\partial}{\partial t}} W_{k} \\
& =e_{\alpha} \cdot \nabla_{e_{\alpha}}\left(\psi^{k} \otimes \nabla_{\frac{\partial}{\partial t}} W_{k}\right)+e_{\alpha} \cdot \psi^{k} \otimes R_{e_{\alpha}, \frac{\partial}{\partial t}} W_{k} \\
& =\not D\left(\psi^{k} \otimes \nabla_{\frac{\partial}{\partial t}} W_{k}\right)+e_{\alpha} \cdot \psi^{k} \otimes R_{\phi_{*}\left(e_{\alpha}\right), \xi}^{N} W_{k} .
\end{aligned}
$$

Please note that here and in the following text $R_{X Y}=-\left[\nabla_{X}, \nabla_{Y}\right]+\nabla_{[X, Y]}$. In conjunction with (2.3), we have

$$
\begin{aligned}
I I & =\int_{M}\left\langle\left.\frac{d}{d t}\right|_{t=0} \psi, \not D \psi\right\rangle+\left\langle\psi,\left.\frac{d}{d t}\right|_{t=0} \not D \psi\right\rangle \\
& =\int_{M}\left\langle\psi, \not D\left(\psi^{k} \otimes \nabla_{\frac{\partial}{\partial t}} W_{k}\right)+e_{\alpha} \cdot \psi^{k} \otimes R_{\phi_{*}\left(e_{\alpha}\right), \xi}^{N} W_{k}\right\rangle \\
& =\int_{M}\left\langle\not D \psi, \psi^{k} \otimes \nabla_{\frac{\partial}{\partial t}} W_{k}\right\rangle+\int_{M}\left\langle\psi^{j}, e_{\alpha} \cdot \psi^{k}\right\rangle\left\langle W_{j}, R_{\phi_{*}\left(e_{\alpha}\right), \xi}^{N} W_{k}\right\rangle \\
& =\int_{M}\left\langle\psi^{j}, e_{\alpha} \cdot \psi^{k}\right\rangle \frac{\left\langle R_{\phi_{*}\left(e_{\alpha}\right), \xi}^{N} W_{k}, W_{j}\right\rangle}{} \\
& =-\int_{M}\left\langle\psi^{j}, e_{\alpha} \cdot \psi^{k}\right\rangle\left\langle R_{W_{j}, W_{k}}^{N} \phi_{*}\left(e_{\alpha}\right), \xi\right\rangle
\end{aligned}
$$

Substituting (2.7) and (2.9) into (2.6) yields

$$
\frac{d L}{d t}=-\int_{M}\left\langle 2 \tau(\phi)+\left\langle\psi^{j}, e_{\alpha} \cdot \psi^{k}\right\rangle R_{W_{j}, W_{k}}^{N} \phi_{*}\left(e_{\alpha}\right), \xi\right\rangle .
$$

Thereby (2.4) follows.

Remark 2.1 The Euler-Lagrange equations of $L$ was firstly derived in [1]. But our denotation is different. 


\section{Zeros of harmonic spinor fields}

In this section, $M$ and $N$ are both oriented Riemannian surfaces. Then $\Sigma M=\Sigma^{+} M \oplus \Sigma^{-} M$, where

$$
\Sigma^{ \pm} M=\left\{\xi \in \Sigma M: \sqrt{-1} e_{1} \cdot e_{2} \cdot \xi= \pm \xi\right\}
$$

(Here $\left\{e_{1}, e_{2}\right\}$ is an orthonormal basis of $T_{\pi(\xi)} M$, and $\pi$ denotes the bundle projection of $\Sigma M$ onto $M$.) In conjunction with $\left(\phi^{-1} T N\right)^{\mathbb{C}}=\phi^{-1}\left(T^{(1,0)} N\right) \oplus \phi^{-1}\left(T^{(0,1)} N\right)$, we have

$$
\begin{aligned}
\Sigma M \otimes\left(\phi^{-1} T N\right)^{\mathbb{C}}= & \left(\Sigma^{+} M \otimes \phi^{-1}\left(T^{(1,0)} N\right)\right) \oplus\left(\Sigma^{+} M \otimes \phi^{-1}\left(T^{(0,1)} N\right)\right) \\
& \oplus\left(\Sigma^{-} M \otimes \phi^{-1}\left(T^{(1,0)} N\right)\right) \oplus\left(\Sigma^{-} M \otimes \phi^{-1}\left(T^{(0,1)} N\right)\right) .
\end{aligned}
$$

Denote by $\pi_{1}^{+}, \pi_{0}^{+}, \pi_{1}^{-}, \pi_{0}^{-}$the projections of $\Sigma M \otimes\left(\phi^{-1} T N\right)^{\mathbb{C}}$ onto the subbundles, respectively. Let $X$ be a tangent vector field on $M$, then $\nabla_{X}$ keeps $\Gamma\left(\Sigma^{ \pm} M\right), \Gamma\left(\phi^{-1}\left(T^{(1,0)} N\right)\right)$ and $\Gamma\left(\phi^{-1}\left(T^{(0,1)} N\right)\right)$ invariant, and $X \cdot \Sigma^{ \pm} M \subset \Sigma^{\mp} M$; therefore

$$
\begin{aligned}
& \not D\left(\Gamma\left(\Sigma^{ \pm} M \otimes \phi^{-1}\left(T^{(1,0)} N\right)\right)\right) \subset \Gamma\left(\Sigma^{\mp} M \otimes \phi^{-1}\left(T^{(1,0)} N\right)\right), \\
& \not D\left(\Gamma\left(\Sigma^{ \pm} M \otimes \phi^{-1}\left(T^{(0,1)} N\right)\right)\right) \subset \Gamma\left(\Sigma^{\mp} M \otimes \phi^{-1}\left(T^{(0,1)} N\right)\right) .
\end{aligned}
$$

Hence $\not D \psi=0$ yields that $\pi_{1}^{+}(\psi), \pi_{0}^{+}(\psi), \pi_{1}^{-}(\psi), \pi_{0}^{-}(\psi)$ are all harmonic spinor fields along $\phi$.

Let $\psi \in \Gamma\left(\Sigma^{+} M \otimes \phi^{-1}\left(T^{(1,0)} N\right)\right)$ be harmonic, we shall derive the equation of $\psi$ in local complex coordinates.

Let $z=x+\sqrt{-1} y, w=u+\sqrt{-1} v$ be complex coordinates of $M, N$, respectively. Then the metric of $M, N$ are of the forms $\lambda(z)|d z|^{2}, \rho(w)|d w|^{2}$, respectively. Denote

$$
s=\left\{e_{1}, e_{2}\right\}, \quad \text { where } e_{1}=\lambda^{-\frac{1}{2}} \frac{\partial}{\partial x}, e_{2}=\lambda^{-\frac{1}{2}} \frac{\partial}{\partial y} .
$$

then $s$ is a local tangent orthonormal frame field; i.e. $s$ is a smooth section of $S O U, U \subset M$. Let $\tilde{s} \in \Gamma(\operatorname{Spin} U)$ be a lift of $s$, i.e. $\eta \circ \tilde{s}=s$. Denote

$$
\psi^{+}=[\tilde{s}, \sigma], \quad \psi^{-}=e_{1} \cdot \psi^{+},
$$

where $\sigma$ is a unit vector in $\Sigma_{2}^{+}$. (The definition of $\Sigma_{2}^{+}$is introduced in [5, Proposition 2.16]). From (3.1),

$$
\begin{aligned}
& e_{1} \cdot \psi^{+}=\psi^{-}, \quad e_{1} \cdot \psi^{-}=-\psi^{+}, \\
& e_{2} \cdot \psi^{+}=\sqrt{-1} \psi^{-}, \quad e_{2} \cdot \psi^{-}=\sqrt{-1} \psi^{+} .
\end{aligned}
$$

And furthermore,

$$
\begin{aligned}
& \frac{\partial}{\partial z} \cdot \psi^{+}=\lambda^{\frac{1}{2}} \psi^{-}, \quad \frac{\partial}{\partial z} \cdot \psi^{-}=0, \\
& \frac{\partial}{\partial \bar{z}} \cdot \psi^{+}=0, \quad \frac{\partial}{\partial \bar{z}} \cdot \psi^{-}=-\lambda^{\frac{1}{2}} \psi^{+} .
\end{aligned}
$$


By the definition of the connection on $\Sigma M$ (see [6, p. 110] and [5, Proposition 4.3]), we have

$$
\begin{aligned}
\not \partial \psi^{+} & =e_{\alpha} \cdot \nabla_{e_{\alpha}} \psi^{+}=\frac{1}{2} e_{\alpha} \cdot\left\langle\nabla_{e_{\alpha}} e_{1}, e_{2}\right\rangle e_{1} \cdot e_{2} \cdot \psi^{+} \\
& =\frac{1}{2}\left\langle\nabla_{e_{1}} e_{1}, e_{2}\right\rangle e_{1} \cdot e_{1} \cdot e_{2} \cdot \psi^{+}+\frac{1}{2}\left\langle\nabla_{e_{2}} e_{1}, e_{2}\right\rangle e_{2} \cdot e_{1} \cdot e_{2} \cdot \psi^{+} \\
& =\frac{1}{2} \frac{\partial \lambda^{-\frac{1}{2}}}{\partial y}(-\sqrt{-1}) \psi^{-}-\frac{1}{2} \frac{\partial \lambda^{-\frac{1}{2}}}{\partial x} \psi^{-} \\
& =-\frac{\partial \lambda^{-\frac{1}{2}}}{\partial \bar{z}} \psi^{-} .
\end{aligned}
$$

Let $f$ be a smooth function on $U$, such that $\psi=f \psi^{+} \otimes \frac{\partial}{\partial w}$, then

$$
\begin{aligned}
0 & =\not D \psi=\not D\left(f \psi^{+} \otimes \frac{\partial}{\partial w}\right) \\
& =\not \partial \psi^{+} \otimes f \frac{\partial}{\partial w}+\frac{2}{\lambda}\left(\frac{\partial}{\partial z} \cdot \psi^{+} \otimes \nabla_{\frac{\partial}{\partial \bar{z}}}\left(f \frac{\partial}{\partial w}\right)+\frac{\partial}{\partial \bar{z}} \cdot \psi^{+} \otimes \nabla_{\frac{\partial}{\partial z}}\left(f \frac{\partial}{\partial w}\right)\right) \\
& =-\frac{\partial \lambda^{-\frac{1}{2}}}{\partial \bar{z}} \psi^{-} \otimes f \frac{\partial}{\partial w}+2 \lambda^{-\frac{1}{2}} \psi^{-} \otimes\left(\frac{\partial f}{\partial \bar{z}} \frac{\partial}{\partial w}+f \nabla_{\left.\frac{\partial w}{\partial \bar{z}} \frac{\partial}{\partial w}+\frac{\partial \bar{w}}{\partial \bar{z}} \frac{\partial}{\partial \bar{w}} \frac{\partial}{\partial w}\right)}\right. \\
& =2 \lambda^{-\frac{1}{2}}\left(\frac{1}{4} \frac{\partial \log \lambda}{\partial \bar{z}} f+\frac{\partial \log \rho}{\partial w} \frac{\partial w}{\partial \bar{z}} f+\frac{\partial f}{\partial \bar{z}}\right) \psi^{-} \otimes \frac{\partial}{\partial w} .
\end{aligned}
$$

Thereby we get the equation of $f$ as follows:

$$
\frac{\partial f}{\partial \bar{z}}+\left(\frac{1}{4} \frac{\partial \log \lambda}{\partial \bar{z}}+\frac{\partial \log \rho}{\partial w} \frac{\partial w}{\partial \bar{z}}\right) f=0 .
$$

From it, we can prove the following proposition.

Proposition 3.1 Let $\psi$ be a harmonic spinor field along $\phi$, which is a smooth map between two oriented Riemannian surfaces. Then $\left|\pi_{1}^{+}(\psi)\right|$ has isolated zeroes and the order of the zeros are well defined, unless $\left|\pi_{1}^{+}(\psi)\right|$ is identically zero. So are $\left|\pi_{0}^{+}(\psi)\right|,\left|\pi_{1}^{-}(\psi)\right|$ and $\left|\pi_{0}^{-}(\psi)\right|$.

Proof As we have seen, $\pi_{1}^{+}(\psi)$ is harmonic whenever $\psi$ is harmonic. Denote $\pi_{1}^{+}(\psi)=$ $f \psi^{+} \otimes \frac{\partial}{\partial w}$, then $f$ satisfies (3.10). Denote

$$
h(z)=\frac{1}{4} \frac{\partial \log \lambda}{\partial \bar{z}}+\frac{\partial \log \rho}{\partial w} \frac{\partial w}{\partial \bar{z}},
$$

then $\frac{\partial f}{\partial \bar{z}}+h f=0$. Let $\zeta$ be a local solution of $\frac{\partial \zeta}{\partial \bar{z}}=h$, then

$$
\frac{\partial\left(f e^{\zeta}\right)}{\partial \bar{z}}=-h f e^{\zeta}+h f e^{\zeta}=0 .
$$

i.e. $f e^{\zeta}$ is holomorphic. Hence the conclusion follows from the well-known fact that the zeros of a holomorphic function are isolated and the order of zeros are well defined, unless it is identically zero. And the proof for $\left|\pi_{0}^{+}(\psi)\right|,\left|\pi_{1}^{-}(\psi)\right|$ and $\left|\pi_{0}^{-}(\psi)\right|$ is similar. 


\section{Weitzenböck-type formulas and Bochner-type formulas}

For a spinor field $\psi$ along a map $\phi: M \rightarrow N$, where $\left(M^{m}, h\right)$ is a Riemannian spin manifold, we can proceed as [1, Proposition 3.4] to have the following Weitzenböck-type formula.

$$
\not D^{2} \psi=-\tilde{\nabla}_{e_{\alpha}} \tilde{\nabla}_{e_{\alpha}} \psi+\frac{1}{4} S \psi+\frac{1}{2} \sum_{\alpha \neq \beta} e_{\beta} \cdot e_{\alpha} \cdot \psi^{j} \otimes R_{\phi_{*}\left(e_{\alpha}\right), \phi_{*}\left(e_{\beta}\right)}^{N} W_{j} .
$$

Here $\psi=\psi^{j} \otimes W_{j},\left\{e_{\alpha}\right\}$ is a local tangent orthonormal frame field on $M$ such that $\nabla e_{\alpha}=0$ at the considered point, and $S$ is the scalar curvature. When $m=2, S=2 K_{M}$, where $K_{M}$ denote the Gauss curvature of $M$, then

$$
\not D^{2} \psi=-\tilde{\nabla}_{e_{\alpha}} \tilde{\nabla}_{e_{\alpha}} \psi+\frac{1}{2} K_{M} \psi+e_{2} \cdot e_{1} \cdot \psi_{j} \otimes R_{\phi_{*}\left(e_{1}\right), \phi_{*}\left(e_{2}\right)}^{N} W_{j} .
$$

Now we assume $M, N$ are both oriented Riemannian surfaces; $\psi \in \Gamma\left(\Sigma^{+} M \otimes \phi^{-1}\right.$ $\left.\left(T^{(1,0)} N\right)\right), \psi \neq 0$ and $\not D \psi=0$. For arbitrary $x \in M$ satisfying $\psi(x) \neq 0$, let $r>0$ such that $\exp _{x}: B(r) \rightarrow M$ is injective; denote $U=\exp _{x}(B(r))$, then we can define a local section of $\Sigma^{+} M$ (denoted by $\psi^{+}$) and a local section of $\phi^{-1}\left(T^{(1,0)} N\right)$ (denoted by $W$ ) on $U$, such that for any geodesic $\gamma$ starting from $x$,

$$
\nabla_{\dot{\gamma}} \psi^{+}=\nabla_{\dot{\gamma}} W=0,
$$

and $\left\langle\psi^{+}, \psi^{+}\right\rangle=1,\langle W, W\rangle=1$; hence at $x$,

$$
\nabla_{e_{\alpha}} \psi^{+}=\nabla_{e_{\alpha}} \nabla_{e_{\alpha}} \psi^{+}=\nabla_{e_{\alpha}} W=\nabla_{e_{\alpha}} \nabla_{e_{\alpha}} W=0 .
$$

$\psi$ could locally be expressed by $\psi=f \psi^{+} \otimes W$, then at $x$,

$$
\tilde{\nabla}_{e_{\alpha}} \tilde{\nabla}_{e_{\alpha}} \psi=\Delta f \psi^{+} \otimes W
$$

Denote $W=\frac{\sqrt{2}}{2}\left(V_{1}-\sqrt{-1} V_{2}\right)$, then $V_{2}=J^{N} V_{1}$, where $J^{N}$ is the complex structure on $N$, and $g\left(V_{i}, V_{j}\right)=\delta_{i j}$. Denote

$$
\phi_{*} e_{i}=\phi_{i j} V_{j}
$$

then

$$
\begin{aligned}
R_{\phi_{*} e_{1}, \phi_{*} e_{2}}^{N} W & =R_{\phi_{11} V_{1}+\phi_{12} V_{2}, \phi_{21} V_{1}+\phi_{22} V_{2}}^{N}\left(\frac{\sqrt{2}}{2}\left(V_{1}-\sqrt{-1} V_{2}\right)\right) \\
& =\operatorname{det}\left(\phi_{i j}\right) R_{V_{1}, V_{2}}^{N}\left(\frac{\sqrt{2}}{2}\left(V_{1}-\sqrt{-1} V_{2}\right)\right) \\
& =\sqrt{-1} J(\phi) K_{N} W .
\end{aligned}
$$

Here $J(\phi)$ denotes the Jacobian of $\phi$ and $K_{N}$ denotes the Gauss curvature of $N$. Substituting (4.3) and (4.4) into (4.2) yields

$$
\begin{aligned}
0 & =\not D^{2} \psi=-\tilde{\nabla}_{e_{\alpha}} \tilde{\nabla}_{e_{\alpha}} \psi+\frac{1}{2} K_{M} \psi+e_{2} \cdot e_{1} \cdot \psi^{+} \otimes R_{\phi_{*} e_{1}, \phi_{*} e_{2}}^{N} W \\
& =\left(-\Delta f+\frac{1}{2} K_{M} f-K_{N} J(\phi) f\right) \psi^{+} \otimes W
\end{aligned}
$$

i.e.

$$
\Delta f=\frac{1}{2} K_{M} f-K_{N} J(\phi) f \quad \text { at } x .
$$


Furthermore,

$$
\begin{aligned}
\Delta|\psi|^{2} & =\Delta|f|^{2}=\bar{f} \Delta f+f \Delta \bar{f}+2|\nabla f|^{2} \\
& =K_{M}|\psi|^{2}-2 K_{N} J(\phi)|\psi|^{2}+2|\nabla \psi|^{2} .
\end{aligned}
$$

From

$$
\begin{aligned}
0 & =e_{1} \cdot \not D \psi=e_{1} \cdot e_{1} \cdot \nabla_{e_{1}} \psi+e_{1} \cdot e_{2} \cdot \nabla_{e_{2}} \psi \\
& =-\left(\nabla_{e_{1}} f\right) \psi^{+} \otimes W+\left(\nabla_{e_{2}} f\right) e_{1} \cdot e_{2} \cdot \psi^{+} \otimes W \\
& =-\left(\nabla_{e_{1}} f+\sqrt{-1} \nabla_{e_{2}} f\right) \psi^{+} \otimes W
\end{aligned}
$$

we have

$$
\nabla_{\bar{Z}} f=0 \text {. }
$$

Here $Z=\frac{\sqrt{2}}{2}\left(e_{1}-\sqrt{-1} e_{2}\right)$ and $\bar{Z}=\frac{\sqrt{2}}{2}\left(e_{1}+\sqrt{-1} e_{2}\right)$, which satisfy $h(Z, \bar{Z})=1$, $h(Z, Z)=h(\bar{Z}, \bar{Z})=0$. Then $\nabla f=\left(\nabla_{Z} f\right) \bar{Z}$ and

$$
|\nabla \psi|^{2}=|\nabla f|^{2}=\left|\nabla_{Z} f\right|^{2}
$$

Furthermore, from

$$
\begin{aligned}
\nabla|\psi|^{2} & =\nabla|f|^{2}=f \nabla \bar{f}+\bar{f} \nabla f \\
& =f\left(\nabla_{\bar{Z}} \bar{f}\right) Z+\bar{f}\left(\nabla_{Z} f\right) \bar{Z}
\end{aligned}
$$

we arrive at

$$
\left.\left.|\nabla| \psi\right|^{2}\right|^{2}=2|f|^{2}\left|\nabla_{Z} f\right|^{2}=2|\psi|^{2}|\nabla \psi|^{2} .
$$

Substituting (4.12) into (4.7) yields

$$
\Delta|\psi|^{2}=K_{M}|\psi|^{2}-2 K_{N} J(\phi)|\psi|^{2}+\frac{\left.\left.|\nabla| \psi\right|^{2}\right|^{2}}{|\psi|^{2}}
$$

at $x \in M$ whenever $\psi(x) \neq 0$.

And at last we derive the following Bochner-type formula

$$
\Delta \log |\psi|=\frac{1}{2} K_{M}-K_{N} J(\phi) .
$$

Similarly, when $\psi \in \Sigma^{+} M \otimes \phi^{-1}\left(T^{(0,1)} N\right), \Sigma^{-} M \otimes \phi^{-1}\left(T^{(1,0)} N\right)$ or $\Sigma^{-} M \otimes \phi^{-1}$ $\left(T^{(0,1)} N\right)$, the corresponding Bochner-type formulas could be derived. We write those results as the following theorem.

Theorem 4.1 Let $M$ and $N$ are both oriented Riemannian surfaces. If $\psi$ is a harmonic spinor field along $\phi: M \rightarrow N$, then $\log \left|\pi_{1}^{+}(\psi)\right|, \log \left|\pi_{0}^{+}(\psi)\right|, \log \left|\pi_{1}^{-}(\psi)\right|$ and $\log \left|\pi_{0}^{-}(\psi)\right|$ satisfy Bochner-type formulas as follows:

$$
\begin{aligned}
& \Delta \log \left|\pi_{1}^{+}(\psi)\right|=\frac{1}{2} K_{M}-K_{N} J(\phi), \\
& \Delta \log \left|\pi_{0}^{+}(\psi)\right|=\frac{1}{2} K_{M}+K_{N} J(\phi), \\
& \Delta \log \left|\pi_{1}^{-}(\psi)\right|=\frac{1}{2} K_{M}+K_{N} J(\phi), \\
& \Delta \log \left|\pi_{0}^{-}(\psi)\right|=\frac{1}{2} K_{M}-K_{N} J(\phi) .
\end{aligned}
$$


When $M$ and $N$ are both compact, since the zeros of $\left|\pi_{1}^{+}(\psi)\right|$ are isolated, there exist a finite number of zeros $p_{1}, \ldots, p_{k}$ in $M$. And similarly the zeros of $\left|\pi_{0}^{+}(\psi)\right|,\left|\pi_{1}^{-}(\psi)\right|$ and $\left|\pi_{0}^{-}(\psi)\right|$ are finite. Integrating both side of (4.15)-(4.18) on $M$, in conjunction with divergence theorem and Gauss-Bonnet formula, we can proceed as [7, pp. 11-12] to get the proposition:

Theorem 4.2 Let $M$ and $N$ are both compact oriented Riemannian surfaces, $\psi$ is a harmonic spinor field along $\phi: M \rightarrow N$. If $\left|\pi_{1}^{+}(\psi)\right|$ is not identically zero, then

$$
\sum_{p \in M,\left|\pi_{1}^{+} \psi\right|(p)=0} n_{p}^{+}=g_{M}-1-\operatorname{deg}(\phi)\left(2 g_{N}-2\right) .
$$

If $\left|\pi_{0}^{+}(\psi)\right|$ is not identically zero, then

$$
\sum_{p \in M,\left|\pi_{0}^{+} \psi\right|(p)=0} m_{p}^{+}=g_{M}-1+\operatorname{deg}(\phi)\left(2 g_{N}-2\right) .
$$

If $\left|\pi_{1}^{-}(\psi)\right|$ is not identically zero, then

$$
\sum_{p \in M,\left|\pi_{1}^{-} \psi\right|(p)=0} n_{p}^{-}=g_{M}-1+\operatorname{deg}(\phi)\left(2 g_{N}-2\right) .
$$

If $\left|\pi_{0}^{-}(\psi)\right|$ is not identically zero, then

$$
\sum_{p \in M,\left|\pi_{0}^{-} \psi\right|(p)=0} m_{p}^{-}=g_{M}-1-\operatorname{deg}(\phi)\left(2 g_{N}-2\right) .
$$

Here $n_{p}^{+}, m_{p}^{+}, n_{p}^{-}, m_{p}^{-}$are respectively the order of $\left|\pi_{1}^{+}(\psi)\right|,\left|\pi_{0}^{+}(\psi)\right|,\left|\pi_{1}^{-}(\psi)\right|,\left|\pi_{0}^{-}(\psi)\right|$ at $p ; \operatorname{deg}(\phi)$ denotes the degree of mapping; $g_{M}$ and $g_{N}$ are genus of $M$ and $N$, respectively.

\section{Proof of main theorems}

In conjunction with Proposition 2.1 and Theorem 4.2, it is not difficult to obtain:

Theorem 5.1 $M$ and $N$ are both compact oriented Riemannian surfaces, and $(\phi, \psi)$ is a Dirac-harmonic map from $M$ to $N$. If $g_{M}=0$ or $\left|g_{M}-1\right|<|\operatorname{deg}(\phi)|\left|2 g_{N}-2\right|$, then $\phi$ has to be a harmonic map.

Proof If $g_{M}=0$ or $\left|g_{M}-1\right|<|\operatorname{deg}(\phi)|\left|2 g_{N}-2\right|$, then $g_{M}-1-\operatorname{deg}(\phi)\left(2 g_{N}-2\right)<0$ or $g_{M}-1+\operatorname{deg}(\phi)\left(2 g_{N}-2\right)<0$. Hence from Theorem 4.2, either $\pi_{1}^{+}(\psi)=\pi_{0}^{-}(\psi)=0$ or $\pi_{0}^{+}(\psi)=\pi_{1}^{-}(\psi)=0$ could be obtained. If $\pi_{1}^{+}(\psi)=\pi_{0}^{-}(\psi)=0$, then there exist smooth functions $f$ and $g$, such that

$$
\psi=f \psi^{+} \otimes \frac{\partial}{\partial \bar{w}}+g \psi^{-} \otimes \frac{\partial}{\partial w},
$$

where the definition of $\psi^{+}, \psi^{-}, \frac{\partial}{\partial w}, \frac{\partial}{\partial \bar{w}}$ is similar to Sect. 3. Hence by (2.4),

$$
\begin{aligned}
\tau(\phi)= & -\frac{1}{2}\left\langle f \psi^{+}, e_{\alpha} \cdot f \psi^{+}\right\rangle R_{\frac{\partial}{\partial w}, \frac{\partial}{\partial \bar{w}}}^{N} \phi_{*}\left(e_{\alpha}\right)-\frac{1}{2}\left\langle g \psi^{-}, e_{\alpha} \cdot g \psi^{-}\right\rangle R_{\frac{\partial}{\partial \bar{w}}, \frac{\partial}{\partial w}}^{N} \phi_{*}\left(e_{\alpha}\right) \\
& -\frac{1}{2}\left\langle f \psi^{+}, e_{\alpha} \cdot g \psi^{-}\right\rangle R_{\frac{\partial}{\partial w}, \frac{\partial}{\partial w}}^{N} \phi_{*}\left(e_{\alpha}\right)-\frac{1}{2}\left\langle g \psi^{-}, e_{\alpha} \cdot f \psi^{+}\right\rangle R_{\frac{\partial}{\partial \bar{w}}, \frac{\partial}{\partial \bar{w}}} \phi_{*}\left(e_{\alpha}\right) \\
= & 0 .
\end{aligned}
$$

So $\phi$ is a harmonic map. When $\pi_{0}^{+}(\psi)=\pi_{1}^{-}(\psi)=0$, the proof is similar. 
It is well known that $S^{2}$ is biholomorphically isomorphic to $\mathbb{C}^{*}=\mathbb{C} \cup\{\infty\}$; hence in the following text we identify $S^{2}$ and $\mathbb{C}^{*}$. Let $h=\lambda(z)|d z|^{2}$ be a metric on $\mathbb{C}^{*} ;$ denote $\tilde{z}=z^{-1}$, then

$$
\lambda(z)|d z|^{2}=\lambda(\tilde{z}(z))|\tilde{z}|^{-4} d \tilde{z}^{2} ;
$$

hence $\lambda(\tilde{z}(z))|\tilde{z}|^{-4}$ is regular at $\tilde{z}=0$; then there exists a constant $c>0$, such that

$$
\lim _{z \rightarrow \infty} \lambda(z)|z|^{4}=c .
$$

And the definition of $e_{1}, e_{2}, \psi^{+}, \psi^{-}$is similar to Sect. 3 .

Theorem 5.2 If $M=S^{2}=\mathbb{C}^{*}$ equipped with metric $h=\lambda(z)|d z|^{2}$ and $N=S^{2}$ equipped with arbitrary metric, $(\phi, \psi)$ is a non-trivial Dirac-harmonic map from $M$ to $N$, then $\phi$ has to be holomorphic or anti-holomorphic, $\psi$ could be written in the form

$$
\psi=e_{\alpha} \cdot \Psi \otimes \phi_{*}\left(e_{\alpha}\right)
$$

and there exist two meromorphic function $u_{1}, u_{2}$ on $\mathbb{C}^{*}$ such that

$$
\Psi=\bar{u}_{1} \lambda^{\frac{1}{4}} \psi^{+}+u_{2} \lambda^{\frac{1}{4}} \psi^{-}
$$

if $u_{i}(i=1$ or 2$)$ has a pole of order $k$ at $z_{0} \in \mathbb{C}$, then $|d \phi|\left(z_{0}\right)=0$ and the order of $|d \phi|$ at $z_{0}$ is no less than $k$; if $\infty$ is a pole of order $k \geq 2$, then $|d \phi|(\infty)=0$ and the order of $|d \phi|$ at $\infty$ is no less than $k-1$. And vice versa.

Proof By Theorem 5.1, $\phi$ has to be a harmonic map. It is well known that when $\operatorname{deg} \phi=0, \phi$ is a constant mapping; when $\operatorname{deg} \phi \geq 1, \phi$ is holomorphic; and when $\operatorname{deg} \phi \leq-1, \phi$ is antiholomorphic (cf. [7, pp. 11-12]). From Theorem 4.2, when $\operatorname{deg} \phi=0,\left|\pi_{1}^{+}(\psi)\right|,\left|\pi_{0}^{+}(\psi)\right|$, $\left|\pi_{1}^{-}(\psi)\right|,\left|\pi_{0}^{-}(\psi)\right|$ are all identically zero, hence $\psi=0$; it is a trivial solution of (2.3) and (2.4). When $\operatorname{deg} \phi \geq 1$, we have $\left|\pi_{0}^{+}(\psi)\right|=\left|\pi_{1}^{-}(\psi)\right|=0$; since $\phi$ is holomorphic, $\phi_{*}\left(e_{1}\right)-\sqrt{-1} \phi_{*}\left(e_{2}\right) \in T^{(1,0)} N, \phi_{*}\left(e_{1}\right)+\sqrt{-1} \phi_{*}\left(e_{2}\right) \in T^{(0,1)} N$, there exist two functions $f, g$ (possibly with isolated singularities), such that

$$
\psi=f \psi^{+} \otimes\left(\phi_{*}\left(e_{1}\right)-\sqrt{-1} \phi_{*}\left(e_{2}\right)\right)+g \psi^{-} \otimes\left(\phi_{*}\left(e_{1}\right)+\sqrt{-1} \phi_{*}\left(e_{2}\right)\right) .
$$

From (3.6), it is easy to obtain

$$
\psi=e_{\alpha} \cdot \Psi \otimes \phi_{*}\left(e_{\alpha}\right)
$$

where $\Psi=g \psi^{+}-f \psi^{-}$. When $\operatorname{deg} \phi \leq-1$, similarly we can construct a spinor $\Psi$ (possibly with isolated singularities) satisfying (5.6).

By [1, Proposition 2.2], $\Psi$ is a twistor spinor, i.e.

$$
\nabla_{v} \Psi+\frac{1}{2} v \cdot \not \partial \Psi=0
$$

for any $v \in T_{p} S^{2}$, where $p$ is an arbitrary regular point of $\Psi$. If $\Psi \in \Gamma\left(\Sigma^{+} M\right)$, then from (3.1), (5.7) is equivalent to

$$
\nabla_{\frac{\partial}{\partial z}} \Psi=0
$$

Denote

$$
\Phi_{0}^{+}=\lambda^{\frac{1}{4}} \psi^{+},
$$


then from

$$
\begin{aligned}
\nabla_{\frac{\partial}{\partial z}} \psi^{+} & =\frac{1}{2}\left\langle\nabla_{\frac{\partial}{\partial z}} e_{1}, e_{2}\right\rangle e_{1} \cdot e_{2} \cdot \psi^{+} \\
& =-\frac{1}{4} \sqrt{-1} \lambda^{\frac{1}{2}}\left\langle\nabla_{e_{1}-\sqrt{-1} e_{2}} e_{1}, e_{2}\right\rangle \psi^{+} \\
& =-\frac{1}{4} \sqrt{-1} \lambda^{\frac{1}{2}}\left(\frac{\partial\left(\lambda^{-\frac{1}{2}}\right)}{\partial y}+\sqrt{-1} \frac{\partial\left(\lambda^{-\frac{1}{2}}\right)}{\partial x}\right) \psi^{+} \\
& =-\frac{1}{4} \frac{\partial \log \lambda}{\partial z} \psi^{+},
\end{aligned}
$$

we have

$$
\nabla_{\frac{\partial}{\partial z}} \Phi_{0}^{+}=\frac{1}{4} \frac{\partial \log \lambda}{\partial z} \lambda^{\frac{1}{4}} \psi^{+}-\frac{1}{4} \frac{\partial \log \lambda}{\partial z} \lambda^{\frac{1}{4}} \psi^{+}=0 .
$$

Let $u_{1}$ be a function on $C^{*}$ (possibly with isolated singularities) such that

$$
\Psi=\bar{u}_{1} \Phi_{0}^{+},
$$

then it is easy to obtain $\frac{\partial u_{1}}{\partial \bar{z}}=0$. Similarly, if $\Psi \in \Gamma\left(\Sigma^{-} M\right)$ is a twistor spinor, then we could obtain

$$
\Psi=u_{2} \Phi_{0}^{-},
$$

where $u_{2}$ is a meromorphic function and $\Phi_{0}^{-}=\lambda^{\frac{1}{4}} \psi^{-}$. Thereby (5.4) follows. The last statement is followed from $\lambda>0$ on $\mathbb{C}$ and (5.2).

On the other hand, let $\psi=e_{\alpha} \cdot \Psi \otimes \phi_{*}\left(e_{\alpha}\right)$, where $\phi$ is holomorphic or anti-holomorphic, and $\Psi$ is a twistor spinor (possibly with isolated singularities) satisfying (5.4). Denote by $z_{1}, \ldots, z_{l} \in \mathbb{C}^{*}$ the singularities of $\Psi$, then it is easily to check that $(\phi, \psi)$ satisfies $(2.3)$ and (2.4) on $\mathbb{C}^{*}-\left\{z_{1}, \ldots, z_{l}\right\}$, and by the assumption on the poles of $u_{i}$ and the zeros of $|d \phi|$, $|\psi|$ is bounded on $\mathbb{C}^{*}-\left\{z_{1}, \ldots, z_{l}\right\}$. Hence the energy of $(\phi, \psi)$ on $\mathbb{C}^{*}$

$$
E\left(\phi, \psi, \mathbb{C}^{*}\right)=\int_{\mathbb{C}^{*}}\left(|d \phi|^{2}+|\psi|^{4}\right)
$$

is finite. The removable singularity theorem (see [1]) yields that $(\phi, \psi)$ is a Dirac-harmonic map from $M$ to $N$.

Acknowledgments The author wishes to express his sincere gratitude to Professor Y.L. Xin in Fudan University, for his inspiring suggestions.

Open Access This article is distributed under the terms of the Creative Commons Attribution Noncommercial License which permits any noncommercial use, distribution, and reproduction in any medium, provided the original author(s) and source are credited.

\section{References}

1. Chen, Q., Jost, J., Li, J., Wang, G.: Dirac-harmonic maps. Math. Z. 254, 409-432 (2006)

2. Chen, Q., Jost, J., Li, J., Wang, G.: Regularity and energy identities for Dirac-harmonic maps. Math. Z. 251, 61-84 (2005)

3. Chen, Q., Jost, J., Wang, G.: Liouville theorems for Dirac-harmonic maps. J. Math. Phys. 48(113517), 13 (2007) 
4. Friedrich, T.: Dirac operators in Riemannian geometry. Graduate Studies in Mathematics, vol. 25. American Mathematical Society, Providence, pp. xvi+195 (2000)

5. Hijazi, O.: Spectral properties of the Dirac operator and geometrical structures. In: Proceedings of the Summer School on Geometric Methods in Quantum Field Theory. 12-30 July 1999, Villa de Leyva, Colombia, World Scientific, Physics (2001)

6. Lawson, H.B., Michelsohn, M.-L.: Spin Geometry. Princeton University Press, Princeton (1989)

7. Schoen, R., Yau, S.T.: Lectures on harmonic maps. Conference Proceedings and Lecture Notes in Geometry and Topology, II. International Press, Cambridge (1997)

8. Xin, Y.L.: Geometry of harmonic maps. Birkhäuser, Cambridge (1996)

9. Zhao, L.: Energy identities for Dirac-harmonic maps. Calc. Var. PDE 28, 121-138 (2007)

10. Zhu, M.: Dirac-harmonic maps from degenerating spin surfaces I: the Neveu-Schwarz case. arxiv: 0803. 3723. 